\title{
The efficacy of a supervised exercise training programme on readmission rates in patients with myocardial ischemia: results from a randomised controlled trial
}

Nuria Santaularia, Josefina Caminal, Anna Arnau, Montserrat Perramon, Jesus Montesinos, Montserrat Abenoza Guardiola and Tiny Jaarsma

\section{Journal Article}

\section{Tweet}

N.B.: When citing this work, cite the original article.

Original Publication:

Nuria Santaularia, Josefina Caminal, Anna Arnau, Montserrat Perramon, Jesus Montesinos, Montserrat Abenoza Guardiola and Tiny Jaarsma, The efficacy of a supervised exercise training programme on readmission rates in patients with myocardial ischemia: results from a randomised controlled trial, European Journal of Cardiovascular Nursing, 2017. 16(3), pp.201212.

http://dx.doi.org/10.1177/1474515116648801

Copyright: SAGE Publications (UK and US)

http://www.uk.sagepub.com/home.nav

Postprint available at: Linköping University Electronic Press

http://urn.kb.se/resolve?urn=urn:nbn:se:liu:diva-136613

LINKÖPINGS

UNIVERSITET 
Authors:

Núria Santaularia

(Department of Rehabilitation) Althaia Xarxa Assistencial Universitària de Manresa, Spain

Josefina Caminal

(Department of Medicine) Universitat Autònoma de Barcelona, Spain

Anna Arnau

(Clinical Research Unit) Althaia Xarxa Assistencial Universitària de Manresa, Spain

Montserrat Perramon

(Department of Cardiology) Althaia Xarxa Assistencial Universitària de Manresa, Spain

Jesus Montesinos

(Clinical Research Unit) Althaia Xarxa Assistencial Universitària de Manresa, Spain

Montserrat Abenoza Guardiola

(Department of Rehabilitation) Althaia Xarxa Assistencial Universitària de Manresa, Spain

Tiny Jaarsma

(Department of Social and Welfare Studies) Faculty of Health Sciences, Linköping University, Sweden

Corresponding author: Núria Santaularia, Department of Rehabilitation, Althaia Xarxa Assistencial Universitària de Manresa, C/Dr.Joan Soler 1-3, 08243 Manresa, Barcelona, Spain

Email: nsantaul@althaia.cat. 


\section{Introduction}

Cardiovascular disease (CVD) is a health problem of the first order all over the world (1). In Europe, CVD is the leading cause of mortality and has a huge impact on the health budget (2). However, postmyocardial ischemia (MI) survival rates have increased in recent years $(3,4)$. A large number of incident MI survivors are discharged from the hospital into the community and are at a high risk for readmission.

In 1964, cardiac rehabilitation was defined for the first time by the World Health Organization (5). Since then the definition has evolved, and cardiac rehabilitation, comprising physical training and education in cardiovascular risk factors, dietary guidance, psychosocial, occupational and physical activity, has been implemented as a treatment for cardiac patients. In 2010, the Cardiac Rehabilitation Section of the European Association of Cardiovascular Prevention and Rehabilitation published a position paper stating that cardiac rehabilitation programs (CRPs) should include appropriate secondary prevention measures as part of a comprehensive multifactorial approach and should apply the standards of European societies

(6). Cardiac rehabilitation programs are also recommended (Class I evidence) by the European Society of Cardiology, the American Heart Association and the American College of Cardiology (7). CRPs provide regular secondary prevention therapies and assessments by a multidisciplinary team of professionals who offer continued education and resolve patients' doubts during the period after MI (8). CRPs decrease mortality and the risk of recurrent $\mathrm{MI}$, promote a healthy lifestyle and improve functional capacity among patients with coronary heart disease $(9,10)$. Studies on post-MI survival, readmissions and physical rehabilitation date back to 1972 (11). Most of these studies agree that physical exercise reduces total mortality (13-87\%) $(9,11-22)$ and cardiovascular mortality $(22-38 \%)(9,12,14-18)$. However, its impact on readmissions has not been conclusively established. Readmission is a valuable indicator of the effectiveness of exercise because it is an indirect measure of clinical stability and of the quality of healthcare delivery. While many studies have found no significant differences in readmissions post-CRP $(9,11,12,14-16,18,21)$, others report that CRP significantly reduced cardiac readmission rates (by between $17 \%$ and $79 \%)(17,19,20,22)$. Moreover, the few studies available on the issue do not focus on identifying the best exercise regimens for reducing readmissions. Despite the acknowledged benefits, the implementation of CRPs varies widely: according to the European Cardiac Rehabilitation Inventory Survey (2008) $(23,24)$, the number of eligible patients admitted to phase II of CRP ranged from below $3 \%$ (in 
Spain) to $90 \%$ (in Lithuania). About half of the countries admitted $\leq 30 \%$ of eligible patients. Therefore, CRPs appear to be underused.

The barriers to referral to CRP need to be overcome (25). Evidence that these programs reduce readmission would provide an additional incentive for hospitals and providers to refer their patients to CRP after MI.

Given the inconsistency of these results, the primary aim of the present study was to evaluate the efficacy of a supervised exercise training program for reducing cardiac readmissions in patients with MI in the first year after hospital discharge. The study also explored differences between groups in all-cause readmission, all-cause death, all-cause hospital emergency visits, functional capacity, quality of life, adherence to cardiac pharmacological treatment, attendance at nurse follow-up visits, satisfaction with care received, and return-to-work.

\section{Methods}

The study protocol of the clinical trial was previously published but a brief summary is described here (26). The trial was registered at Current Controlled Trials as ISRCTN57634424, dated 30/04/2010.

\section{Ethical approval}

The protocol had been approved on 30 June 2009 by an independent Ethical Committee of Clinical Research (Comitè d’ètica d'Investigació Clínica de la Fundació Unió Catalana d’Hospitals) chaired by Dra. Imma Guasch Jordan, with the protocol number CEIC Code 09/38. This study complied with the sixth version of the Declaration of Helsinki.

Study design

Open, controlled, randomized, hospital-based clinical trial including patients admitted to the Cardiology Department for MI. 
Study subjects

The inclusion criteria were: age over 18, diagnosis of MI (myocardial ischemia, pre-infarct angina, angina pectoris, other specific forms of chronic ischemic heart disease or unspecified ischemic heart disease) during the current admission, residence in the catchment area of our hospital, absence of cognitive deficit (Pfeiffer test: 0-2 mistakes), sufficient functional capacity to follow the CRP (Barthel index >60), and willingness to participate in the study and to provide signed informed consent. Patients were excluded if they had symptoms of right heart failure producing pulmonary hypertension or dyspnea caused by severe pulmonary pathology, additional comorbidities affecting the prognosis of cardiac disease, major comorbidities or limitations that could interfere with the exercise training program.

\section{Randomization}

Patients were randomly assigned to the control or the intervention group. Patients in the intervention group were included in the supervised exercise-training program, while controls received standard care. A randomization list in blocks of 10 was created by a computer random number generator. The randomization list and the allocation of patients to each group were independently controlled by the Clinical Research Unit.

\section{Study arms}

Control. Patients assigned to the control group received the standard care given at the hospital. During hospitalization, the cardiac nurse or the physiotherapist assessed the cardiovascular risk factors and other clinical variables in each patient and gave oral and written information on cardiovascular risk factors. Hospitalized patients were instructed to do exercises in order to regain mobility and to maintain muscle tone and peripheral circulation, and were taught breathing exercises by the physiotherapist to improve their breathing patterns. Before discharge, the physiotherapist provided patients with guidance on how to return to physical activity. After hospital discharge, patients were scheduled for follow-up visits with a cardiac nurse at the first month, and 3, 6 and 12 months (visits 1, 2, 3 and 4 respectively) after hospital 
discharge in order to control the risk factors, reinforce the education measures for disease control and review their adherence to cardiac medication and CRP follow-up.

Intervention. In addition to standard care, patients randomized to the intervention group underwent a supervised outpatient exercise training program, based on the results of the exercise stress test and bearing in mind the physical limitations imposed by their co-morbid conditions. The intervention started within three days of the exercise stress test, which was performed one month after hospital discharge. The program was performed in the hospital. It comprised three hours a week (spread over three alternate days) of supervised exercise training, for a 10-week period. Exercise classes were supervised by a physiotherapist and consisted of 10 minutes of warm-up and muscle stretching, 30 minutes of aerobic exercises on a cycloergometer, 15 minutes of isotonic exercises for the upper and lower limbs and five minutes of cool-down. Moreover, instructions were given on self-pulse counting, the use of the Borg scale (a perceived exertion scale which is a numerical value of a person's perception of the intensity of the effort made during physical activity), relaxation exercises, breathing techniques, postural health and minimizing physical effort. Aerobic exercise intensity was between $75-90 \%$ of the maximum heart rate obtained in the previous exercise stress test and progressed according to the perceived exertion rate (1115). Resistance training was performed with 10-15 repetitions for three sets, maintaining a perceived exertion rate of 11-14. The physiotherapist checked that patients were exercising at their prescribed intensity with a pulse oximeter (Quirumed ${ }^{\circledR}$ Health \& Care).

\section{Procedure}

Patients were selected after assessment of the clinical history and consultation with the cardiologist. In the inpatient setting, eligible patients were given information (both verbal and written) related to the study and their doubts were discussed and resolved. Before discharge (visit -1 ), researchers obtained written informed consent from eligible patients who agreed to participate. On discharge (visit 0) we contacted the Clinical Research Unit to discuss the randomization process. Patients attended four cardiac nurse visits (visits 1, 2, 3 and 4). During visits -1 and 1, the cardiac nurse recorded baseline variables. Readmissions and length of hospital stay for cardiac disease, readmissions and length of hospital stay for all causes, all-cause death, all-cause hospital emergency visits and attendance at nurse follow-up visits 
were recorded over a 12-month follow-up period. Functional capacity was evaluated at baseline and at visit 2. Quality of life (EuroQuol5) was recorded at baseline and at visits 2 and 4 . Adherence to cardiac pharmacological treatment, satisfaction with care received and return-to-work were recorded at visit 4. A detailed procedure was included in the published protocol study (26).

\section{Main outcomes}

The main outcomes included the percentage of patients readmitted, length of hospitalization for cardiac disease during the first year after hospital discharge, and time to first hospital readmission for cardiac disease. An independent committee blind to the patient's treatment group assessed the main outcomes. This committee comprised a cardiologist, a rehabilitation cardiologist and a health information manager, all from different centers.

\section{Secondary outcomes}

Secondary outcomes were the percentage of patients readmitted and length of hospitalization for all causes during the first year after hospital discharge, all-cause death, all-cause hospital emergency visits, functional capacity (maximum double product, chronotropic index, energy expenditure and time of exercise performed were retrieved from the exercise stress test), quality of life (EuroQol5 (27)), adherence to cardiac pharmacological treatment (Haynes-Sackett test (28)), attendance at nurse follow-up visits, satisfaction with care received (questions selected from a questionnaire devised by the local public health department (29)) and self-reported return-to-work.

\section{Statistical analysis}

To ensure a statistical power of $80 \%$ in order to detect differences through a two-tailed chi-square test for two independent samples, with a level of significance of 0.05 , and assuming proportions of cardiac readmissions of $25 \%$ in the control group and $12 \%$ in the intervention group, each group had to comprise 139 patients. Assuming a 5\% loss to follow-up, we estimated that 146 patients would be needed in each arm. 
Descriptive statistics were used to characterize the sample. Means and standard deviations were used for continuous variables and frequencies with percentages for categorical variables. We tested for significant differences between the baseline characteristics of the control and intervention groups. For continuous variables, we used the Student's t-test or the non-parametric Mann-Whitney $U$ test. We used the $\chi^{2}$ test to compare categorical variables or Fisher's exact test or bilateral exact p-value in contingency tables where the expected frequencies were below than five. The percentage of patients readmitted for cardiac disease was analyzed through a two-tailed $\chi^{2}$ test for two independent samples, and time to first hospital readmission for cardiac disease was analyzed as time to event with Kaplan-Meier estimation and Cox proportional regression models. We used the log-rank test to compare the survival curves. We reported the unadjusted and adjusted hazard ratios (HR) and $95 \%$ confidence intervals $(95 \% \mathrm{Cl})$.

To assess the effect on quality of life from a longitudinal perspective, a marginal Generalized Estimating Equations model with the logit as link function was applied in order to estimate the population-average effects. An exchangeable working correlation matrix was assumed. The models were validated via insample k-folding cross validation.

A two-sided $\alpha$-level of 0.05 was considered statistically significant. Data were analyzed using IBM SPSS Statistics for Windows v.20, Stata v.10 and R version 3.0.2.

\section{Results}

Between June 2010 and June 2012, 478 patients were evaluated for eligibility, of whom 86 were randomized. Two hundred sixty-nine did not meet the inclusion criteria (56\%) while 111 (23\%) refused to participate and 12 (3\%) for other reasons. The most common reason for exclusion was the presence of additional comorbidities affecting the prognosis of cardiac disease, and other comorbidities or limitations that interfered with the exercise training program. Eighty-six patients ( $41 \%$ of the patients who met the inclusion criteria) agreed to participate and provided written informed consent. No patients were lost to follow-up. One patient in the intervention group who withdrew prior to the baseline assessment was excluded from the analysis (see Figure 1). 
No statistically significant differences in all baseline characteristics were observed between patients who agreed to participate and those who did not in any of the variables except age, which was higher in the non-participants (65 vs 60; $p=0.006$ ) (see Appendix 1 , supplementary data).

\section{Baseline characteristics}

Table 1 shows the baseline characteristics of the 44 patients in the control group and the 41 patients in the intervention group. No significant differences between groups were observed, with the exception of gender: $23 \%$ of the control group were women, compared with $7 \%$ in the intervention group $(p=0.049)$. Mean age was 60 years (SD 11). Myocardial ischemia was the principal cause of referral. Most patients were overweight and had a mean waist circumference of $99 \mathrm{~cm}$. Smoking was reported by $14 \%$ of the control group and by $7 \%$ of the intervention group. Hypertension was recorded in $52 \%$ of controls and $44 \%$ of the intervention group, and $46 \%$ of controls and $49 \%$ of the intervention group had family history of heart disease.

\section{Main outcomes}

Readmission rates during the first year for cardiac disease were $14 \%(95 \% \mathrm{Cl}: 5-27)$ in the control group and $5 \%(95 \% \mathrm{Cl}: 1-17)$ in the intervention group $(p=0.268)$. In the control group the median length of hospital stay of readmissions for cardiac disease was 2 days [p25-p75; 1-4] and 7 days [p25-p75; 6-8] $(p=0.145)$ in the intervention group (see Table 2$)$. The likelihood of readmission for cardiac diseases during the year of follow-up was higher in the control group (log rank test $p=0.1671$ ) (see Figure 2 ) with a crude HR of 0.34 (95\% Cl: 0.07-1.69). The hazard ratio in the intervention group adjusted for the gender variable was 0.32 (95\% Cl: 0.06-1.61).

\section{Secondary outcomes}

All-cause readmission rates were $23 \%(95 \% \mathrm{Cl}: 12-38)$ in the control group and $15 \%(95 \% \mathrm{Cl}: 6-29)$ in the intervention group $(p=0.34)$. There were no deaths during the first year of follow-up in either group. In the control group, $50 \%$ of patients $(95 \% \mathrm{Cl}: 35-65)$ were treated once or more in the hospital emergency service, compared with $24 \%$ (95\% Cl: $12-40)$ of patients in the intervention group $(p=0.015)$. 
The baseline functional capacity according to the exercise stress test and the change between the assessments at baseline and at three months are shown in Table 3. This table shows the information on the 38 patients in the control group and the 40 patients in the intervention group who performed the exercise stress test at baseline and at 3 months. At three months, significant increases in the maximum double product $(p=0.016)$ and the chronotropic index $(p=0.006)$ were observed in the intervention group. Energy expenditure and time of exercise performed were similar in the two groups.

The results for quality of life during follow-up showed that the proportion of patients reporting mobility problems rose in the control group, but fell in the intervention group. No significant differences were observed between groups for the rest of the dimensions (Appendix 2, supplementary data).

Adherence to cardiac pharmacological treatment was $89 \%$ in the control group and $95 \%$ in the intervention group and attendance at nurse follow-up visits was $100 \%$ and $95 \%$ respectively. Regarding satisfaction with the care received, the mean score was $9 / 10$ in both groups. In the control group $36 \%$ of patients returned to work compared to $77 \%$ of patients in the intervention group (table 2 ).

\section{Discussion}

The supervised physical exercise program described here significantly reduced the number of patients seen at the hospital emergency service, and improved rates of return-to-work and the functional capacity. We also found that patients who participated in the program were readmitted less often than patients in the control group (5 vs 14\%). Unfortunately, the fact that we did not reach the target sample size meant that the study lacked the statistical power required to detect statistically significant differences between the groups, although all the results reflected better performance in the intervention group.

Several systematic reviews and meta-analyses confirm that exercise-based cardiac rehabilitation reduces all-cause and cardiac death $(9,19)$ but does not clearly modify the risk of recurrent MI. There were no deaths in either study group. This may have been due to our use of very restrictive inclusion criteria. Although we did not measure the recurrence of $\mathrm{Ml}$ as such, we looked at readmission rate after cardiac rehabilitation, which might be seen as indicative of recurrent MI. However, the lack of homogeneity in the variables assessed in the studies - fatal MI, non-fatal MI, all-cause readmission or cardiac readmission - makes comparisons difficult. In two meta-analysis $(9,18)$ it was concluded that neither exercise-only nor 
comprehensive cardiac rehabilitation had any effect on the recurrence of non-fatal MI. However, a third meta-analysis (20) found that secondary prevention programs were able to reduce rates of recurrent $\mathrm{MI}$ by $17 \%$ after 12 months. Programs based on education and counseling with a structured exercise component showed a significant reduction in $\mathrm{MI}$ recurrence of $38 \%$ while programs that included risk factor education or counseling without a structured exercise component, or programs that were solely exercise-based, did not achieve significant changes. Our study was designed to compare the rates of readmission due to cardiac disease obtained by a supervised physical exercise program and by standard care, which, in our case, includes risk factor education and counseling in all patients. Although the differences were not significant, in the intervention group we observed a reduction in the risk of cardiac readmission of around $66 \%$. The objectives pursued by this project have a direct impact on the welfare of patients with MI. Although there have been significant increases both in the number of rehabilitation programs in Spain during the last decade and in the number of patients treated, we still lag behind other countries in Europe. The ESRECA study (30) noted the scarcity, and the heterogeneity, of the human and material resources available and especially of educational, psychological and physical exercise interventions. During admission and follow-up, patients and their families received detailed recommendations regarding lifestyle habits, physical exercise and other aspects to consider in order to favor their active control of the disease and to increase safety, which are both highly important factors for reducing the number of readmissions. From the standpoint of health planning and management, the findings of the study may allow substantial savings in the health service budgets by helping to lower readmission rates and shorten hospital stay in a disease as frequent as MI. Although the efficacy and efficiency of CRP seems obvious, their implementation in our country remains low. The ESRECA study (30) concluded that the institutions themselves were to blame for the low level of development of these programs in Spain.

Another interesting finding to add to the existing literature is that patients in the intervention group performed better exercise stress tests in the three months of follow-up than the control group, since they recorded higher maximum double product and chronotropic index values. The data obtained confirm previous studies showing that physical exercise improves activity tolerance (31). This engagement in 
physical activity may also account for the significant improvement in patients' mobility in the intervention group over time.

Adherence to cardiac pharmacological treatment was relatively high in both groups, above the figure of $50 \%$ reported in the literature (32). This positive result may have been due to the nurse follow-up visits in both groups in which patients received encouragement from the nursing staff and were able to discuss and resolve any doubts regarding the medical protocol. Previous studies have reported drop-out rates from nurse follow-up visits in the first year to be between $50 \%$ and $90 \%(33,34)$. The high adherence observed in both our study groups may be due to the fact that, during hospitalization $(35,36)$, information regarding the CRP and cardiovascular risk factors is given "face to face" (37) to patients and their families, and also because the CRP is initiated relatively quickly. We should also consider the possible influence of the Hawthorne effect deriving from inclusion in an experimental study. Patients' satisfaction with the care received was also very high in both groups.

The results obtained corroborate those of previous studies which have reported that supervised physical exercise programs accelerate return-to-work during the first year of follow-up $(38,39)$ and demonstrate that CRPs can contribute to preserving or recovering an active social life (40). Reducing cardiac readmissions and improving the quality of life and functional capacity of the patient accelerates return to normal life and shortens convalescence time, and thus helps to reduce the direct and indirect costs arising from occupational disability.

\section{Limitations}

One of the main limitations of the study is that we did not reach the sample size initially intended. This might have been due to the highly restrictive inclusion criteria, since $51 \%$ of screened patients were excluded for presenting a comorbidity that would affect the prognosis of the MI. The patients who declined to participate in the program expressed reasons very similar to those mentioned in the literature: the impossibility of adapting to the protocol, transport difficulties, living at a considerable distance from the rehabilitation center, reluctance to abandon behaviors associated with cardiovascular risk factors and the fact that they were already performing tests at other health centers $(41,42)$. 
The large catchment area of our hospital may have aggravated the difficulties of recruitment. Furthermore, recruitment was slower than expected, so the inclusion period was expanded until a maximum of two years in order to ensure that the execution of the study was not altered by factors such as changes in health organization, and so on. Despite these shortcomings, we feel that our study of the effects of a supervised exercise program organized as part of our routine clinical practice offers an accurate reflection of practices in the real world.

In line with previous studies $(41,43,44)$, the patients who declined to participate $(56 \%)$ were significantly older than those who agreed; the decliners also comprised a higher proportion of retirees, more patients of pyknic body type, and presented a higher percentage of cardiovascular risk factors (high blood pressure, diabetes mellitus and family history of heart disease). This suggests that the rate of readmissions recorded might have been higher if less strict selection criteria had been applied.

Both groups were instructed to exercise during hospitalization and all patients received information on the importance of exercising after discharge. Additionally the intervention group participated in a supervised outpatient exercise training program. A cross-over effect may have affected the internal validity; that is, the control group may have obtained better results for functional capacity and thus reduced the differences in relation to the intervention group. To control for this potential confounder, all patients completed a physical questionnaire (Capersen and Powell classification) at follow-up visits. No differences in the percentage of patients performing regular physical activity were observed between the two groups (data not shown).

The fact that the groups were unbalanced in terms of gender may have affected the main and secondary outcomes. However, stratification of the statistical analysis by gender showed the same results for men as for the entire sample, both for cardiac readmissions (the main endpoint of the study), and for secondary outcomes such as all-cause readmissions, all-cause hospital emergency visits, stress test or return to work (data not shown).

Considering the nature of the intervention (cardiac rehabilitation) an open trial was unavoidable and the blinding of the subjects and therapists was not possible. This may have affected the outcomes since patients in the control group might have been disappointed not to be randomized to rehabilitation; 
equally, it may be that the intervention patients gave more positive answers. However, the endpoint committee who assessed the primary outcomes was blinded for group assignment.

\section{Recommendations for future studies}

This study has assessed the benefits obtained by a CRP incorporating supervised physical exercise in a large geographical health area in which patients may live at significant distances from their health centers. The results highlight the need to ensure equitable access to programs run by multidisciplinary teams who are experts in cardiology and rehabilitation, so it would be interesting to systematize the implementation of this program in similar health regions. To be able to assess the effects of a supervised exercise training program on readmission it is necessary to optimize patient recruitment: for example, by providing transport from home to the rehabilitation center, or creating strategies to bring the programs to patients instead of bringing patients to programs. At the same time, the definition of readmission needs to be standardized to enable proper comparison of studies. Another new area which we see as particularly important is the creation of new strategies that favor accessibility, such as telemedicine.

Funding

This study was supported by a grant from the Col.legi de Fisioterapeutes de Catalunya (no: R01/08-09).

\section{Acknowledgements}

We are particularly grateful to Jordi Balcells Iranzo and Enric Vers Soto for their technical assistance. We thank Michael Maudsley of the Language Service of the University of Barcelona for his help with the English and Àngel Ruiz for performing the statistical analysis of quality of life.

\section{Conflict of interest}

None declared. 


\section{References}

(1) World Heart Federation. World Health Statistics 2008. 2008, www.world-heartfederation.org/press/news/detail/article/world-health-statistics-2008/ (2008, accessed 5 May 2012).

(2) Nichols M, Townsend N, Luengo-Fernandez R, et al. European Cardiovascular Disease Statistics 2012. Ed.Brussels: European Heart Network, 2012.

(3) Law MR, Watt HC, Wald NJ. The underlying risk of death after myocardial infarction in the absence of treatment. Arch Intern Med 2002;162: 2405-2410.

(4) Neal B. Secondary prevention of cardiovascular disease: fixed dose combinations. In: Kaplan W, Laing R (eds) Priority medicines for Europe and the world. Geneva: World Health Organisation, 2004.

(5) Brown RA. Rehabilitation of patients with cardiovascular diseases. Report of a WHO Expert Committee. World Health Organ Tech Rep Ser 1964; 270: 3-46.

(6) Piepoli M, Corràs U, Benzer W, et al. Secondary prevention through cardiac rehabilitation: from knowledge to implementation. A position paper from the Cardiac Rehabilitation Section of the European Association of Cardiovascular Prevention and Rehabilitation. Eur J Cardiovasc Prev Rehabil 2010; 17: 1-17.

(7) Corrà U, Piepoli MF, Carré F, et al. Secondary prevention through cardiac rehabilitation: physical activity counselling an d exercise training: key components of the position paper from the Cardiac Rehabilitation Section of the European Association of Cardiovascular Prevention and Rehabilitation. Eur Heart J 2010; 31: 1967-1974.

(8) Dunlay SM, Pack QR, Thomas RJ, et al. Participation in cardiac rehabilitation, readmissions, and death after acute myocardial infarction. Am J Med 2014; 127: 538-546.

(9) Taylor R, Brown A, Ebrahim S, et al. Exercise-Based Rehabilitation for Patients with Coronary Heart Disease: Systematic Review and Meta-analysis of Randomized Controlled Trials. Am J Med 2004; 116: 682692.

(10) Heran BS, Chen JM, Ebrahim S, et al. Exercise-based cardiac rehabilitation for coronary heart disease. Cochrane Database Syst Rev 2011; 7: 1-91.

(11) West R. Evaluation of cardiac rehabilitation programmes. In: BMJ Publishing Group (eds) Cardiac Rehabilitation. London, 1995, pp. 184-206.

(12) Shaw LW. Effects of a prescribed supervised exercise program on mortality and cardiovascular morbidity in patients after myocardial infarction. The National Exercise and Heart Disease Project. Am J Cardiol 1981; 48: 39-46.

(13) May G, Eberlein K, Furberg C, et al. Secondary Prevention After Myocardial Infarction: A Review of Long-Term Trials. Prog Cardiovasc Dis 1982; 24: 331-352.

(14) Oldridge NB, Guyatt GJ, Fischer ME, et al. Cardiac rehabilitation after myocardial infarction. Combined Experience of Randomized Clinical Trials. JAMA 1988; 260: 945-950.

(15) O'Connor GT, Buring JE, Yusuf S, et al. An overview of randomized trials of rehabilitation with exercise after myocardial infarction. Circulation 1989; 80: 234-244.

(16) Bobbio M. Does myocardial infarction rehabilitation prolong survival? A meta-analytic survey. G Ital Cardiol 1989; 19: 1059-1067. 
(17) Hedback B, Perk J and Wodlin P. Long-term reduction of cardiac mortality after myocardial infarction: 10-year results of a comprehensive rehabilitation programme. Eur Heart J 1993; 14: 831-835.

(18) Jolliffe J, Rees K, Taylor RRS, et al. Exercise-based rehabilitation for coronary heart disease (Review). Cochrane Database Syst Rev 2001.

(19) Boulay P and Prud'homme D. Health-care consumption and recurrent myocardial infarction after 1 year of conventional treatment versus short- and long-term cardiac rehabilitation. Prev Med 2004; 38: 586-593.

(20) Clark A, Harting L, Vandermeer B, et al. Meta-Analysis: Secondary Prevention Programs for Patients with Coronary Artery Disease. Ann Intern Med 2005; 9: 659-672.

(21) Nielsen KM, Faergeman O, Foldspang A, et al. Cardiac rehabilitation: Health characteristics and socioeconomic status among those who do not attend. Eur J Public Health 2008; 18: 479-483.

(22) Canyon S and Meshgin N. Cardiac rehabilitation - reducing hospital readmissions through community based programs. Aust Fam Physician 2008; 37: 575-577.

(23) Bjarnason-Wehrens B, McGee H, Zwisler AD, et al. Cadiac rehabilitation in Europe: results from the European Cardiac Rehabilitation Inventory Survey. Eur J Cardiovasc Prev Rehabil 2010; 17: 410-418.

(24) Leon-Latre M, Mazon-Ramos $\mathrm{P}$, Marcos E, et al. Update on cardiovascular prevention and cardiac rehabilitation. Rev Esp Cardiol 2009; 62: 4-13.

(25) Brown TM, Hernandez AF, Bittner V, et al. Predictors of cardiac rehabilitation referral in coronary artery disease patients: findings from the American Heart Association's Get With The Guidelines Program. J Am Coll Cardiol 2009; 54: 515-521.

(26) Santaularia N, Caminal J, Arnau A, et al. Randomized clinical trial to evaluate the effect of a supervised exercise training program on readmissions in patients with myocardial ischemia: a study protocol. BMC Cardiovasc Disord 2013; 13: 1-8.

(27) Badia X, Roset M, Montserrat S, et al. The Spanish version of EuroQol: a description and its applications. European Quality of Life scale. Med Clin 1999; 1: 79-85.

(28) Haynes RB, Sackett DL and Taylor DW. How to detect and manage low patient compliance in chronic illness. Geriatrics 1980; 35 :91-93.

(29) CatSalut. Enquesta d'Atenció Especialitzada Ambulatòria 2008, www20.gencat.cat/docs/salut/Minisite/catsalut/Ciutadania/serveis atencio salut/Documents/Arxius/0 8 aea questionari.pdf (2008, accessed 7 December 2013).

(30) García Hernández P, Álvarez Leiva MI, Martínez Castellanos T, et al. Estudio ESRECA. Situación actual de los programas de rehabilitación cardíaca en España. Enfermería en Cardiología 2014; 62:76-84.

(31) Gassner L, Dunn S and Piller N. Aerobic exercise and the post myocardial infraction patient: A review of the literature. Heart Lung 2003; 32: 258-265.

(32) Sabate E. Adherence to long-term therapies: evidence for action, www.who.int/chp/knowledge/publications/adherence_report/en/ (2013, accessed 23 November 2013).

(33) Carlson JJ, Johnson JA, Franklin BA, et al. Program participation, exercise adherence, cardiovascular outcomes, and program cost of traditional versus modified cardiac rehabilitation. Am J Cardiol 2000; 86: 17-23. 
(34) Arthur HM, Smith KM, Kodis J, et al. A Controlled trial of hospital versus home-based exercise in cardiac patients. Med Sci Sports Exerc 2002; 34: 1544-1550.

(35) Braun LT. Cardiac rehabilitation: moving from "best kept secret" to best practice. J Cardiovasc Nurs 2008; 23: 482-483.

(36) French DP, Cooper A and Weinman J. Illness perceptions predict attendance at cardiac rehabilitation following acute myocardial infarction: a systematic review with meta-analysis. J Psychosom Res 2006; 61: 757-767.

(37) Arena R, Williams M, Forman DE, et al. Increasing Referral and Participation Rates to Outpatient Cardiac Rehabilitation: The Valuable Role of Healthcare Professionals in the Inpatient and Home Health Settings. Circulation 2012; 30: 1-9.

(38) Maroto J, Armengol X, Artigao R, et al. Rehabilitación del paciente coronario. Prevención secundaria. 2006.

(39) Lee AJ, Strickler GK and Shepard DS. The economics of cardiac rehabilitation and lifestyle modification: A review of literature. J Cardiopulm Rehabil Prev 2007; 27:135.

(40) Gómez-González A, Montiel-Trujillo A, Bravo-Escobar R, et al. Equipo multidisciplinario en las Unidades de Rehabilitación Cardíaca. ¿Qué papel desempeñamos? Rehabilitación 2006; 40: 290-300.

(41) Fernandez RS, Davidson P and Griffiths R. Cardiac rehabilitation coordinators' perceptions of patientrelated barriers to implementing cardiac evidence-based guidelines. J Cardiovasc Nurs 2008;23: 449-457.

(42) Petter M, Blanchard C, Kemp KA, et al. Correlates of exercise among coronary heart disease patients: review, implications and future directions. Eur J Cardiovasc Prev Rehabil 2009; 16: 515-526.

(43) Comoss P. Improving utilization of cardiac rehabilitation services: where to start? J Cardiovasc Nurs 2008; 23: 480-481.

(44) Davies P, Taylor F, Beswick A, et al. Promoting patient uptake and adherence in cardiac rehabilitation. Cochrane Database Syst Rev 2010; 7:1-39. 